\title{
Microstructure Characterization of TRIP Steel CMnAlSi
}

\author{
Prof. Andrzej Lis \\ Częstochowa University of Technology \\ Institute of Materials Science and Engineering, \\ Armii Krajowej 19, \\ 42-201 Częstochowa, Poland \\ lis@wip.pcz.pl
}

Abstract - Finely grained multiphase microstructures of TRIP- aided steel CMnAISi were characterized by optical microscopy, scanning microscopy and atomic force microscopy observation.

Keywords- multiphase steel, TRIP (Transformation Induced Plasticity), SEM (Scanning Electron Microscopy), AFM (Atomic Force Microscopy)

\section{INTRODUCTION}

One of the greatest achievements of the contemporary metallurgy was development of modern materials for motorization such as multiphase TRIP type steel. Microstructure of Transformation Induced Plasticity steel consists of ferrite - bainite matrix and residual austenite and martensite islands. That type of microstructure is characterized by good combination of strength and plasticity [1]

\section{RESULTS AND DISSCUSION}

The heat treatment was done on an mild-alloy low carbon CMnAlSi steel with chemical composition as follows: $0,15 \% \mathrm{C}, 1,55 \% \mathrm{Mn}, 1,01 \% \mathrm{Si}, 1,09 \% \mathrm{Al}, 0,013 \% \mathrm{P}, 0,006 \%$ $\mathrm{S}$ and $0,003 \% \mathrm{~N}$. Intercritical annealing was performed at $800^{\circ} \mathrm{C} / 600$ s to obtain a two-phase structure of ferrite and austenite. Then sample was cooled down at a rate $5^{\circ} \mathrm{C} / \mathrm{s}$ to room temperature. Phase transformation diagram on cooling from $800^{\circ} \mathrm{C}$ was established. It is shown in Figure 1. The dilatometer DIL 805 A/D was used for time temperature transformation measurements. After continuous cooling dilatometry samples were polished and etched in nital and observed on optical microscope Axiovert 25 with digital attachment Zeiss. Then observations with scanning electron microscope JSM-6610 LM were performed. The scope of research included topographical analysis of the surface with the use of atomic force microscope Veeco Multimode with NanoScope V controller by half-contact method gathering data from certain height, amplitudes of vibration and phase contact. Surface pictures were taken for areas of $1-10 \mu \mathrm{m}^{2}$ in a two-dimension (2D) scale.

\author{
MSc Magda Dryja, PhD Paweł Wieczorek \\ Czestochowa University of Technology \\ Institute of Materials Science and Engineering, \\ Armii Krajowej 19, \\ 42-201 Częstochowa
}

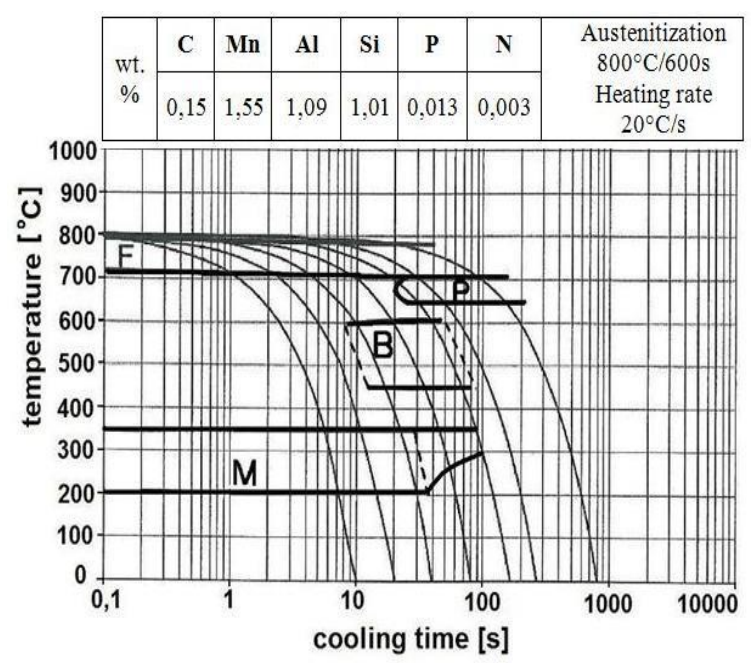

Figure 1. Time Temperature Transformation Diagram for CMnAlSi steel

The final microstructure was composed by the phases: ferrite and martensite or bainite. At cooling rate smaller than $3{ }^{\circ} \mathrm{C} / \mathrm{s}$ some areas of pearlite were observed. Typical TRIP microstructure after cooling at $5^{\circ} \mathrm{C} / \mathrm{s}$ from $(\alpha+\gamma)$ region was shown in Figure 2 and Figure 3.

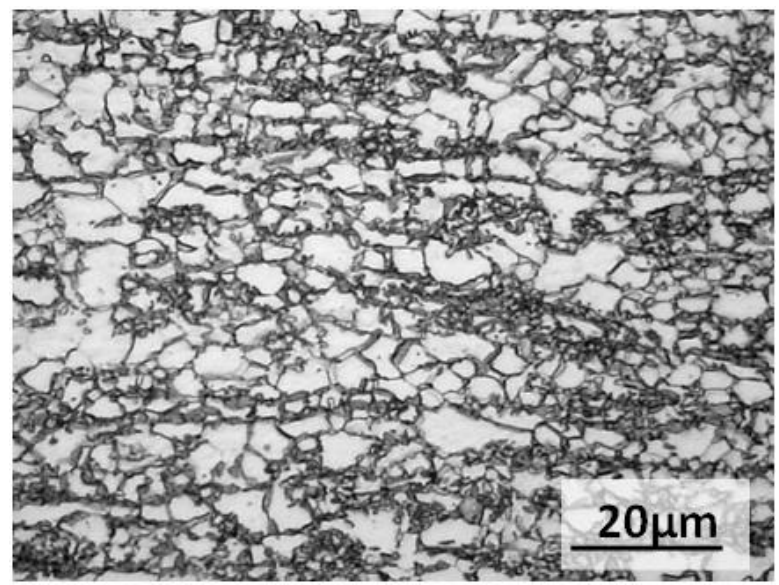

Figure 2. TRIP microstructure with optical microscope of CMnAlSi steel 


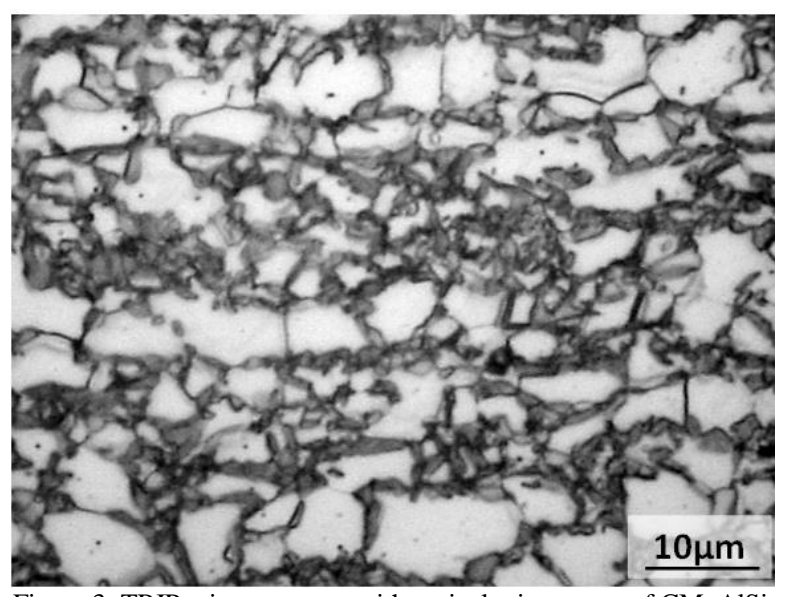

Figure 3. TRIP microstructure with optical microscope of CMnAlSi

More detailed study of topography of grain-refined microstructure was achieved by Scanning Electron Microscopy investigation Figure 4 and Figure 5. Small colonies of the pearlite with very fine lathes of cementite are shown in Figure 4 and small islands of martensite lathes are presented in Figure 5 .

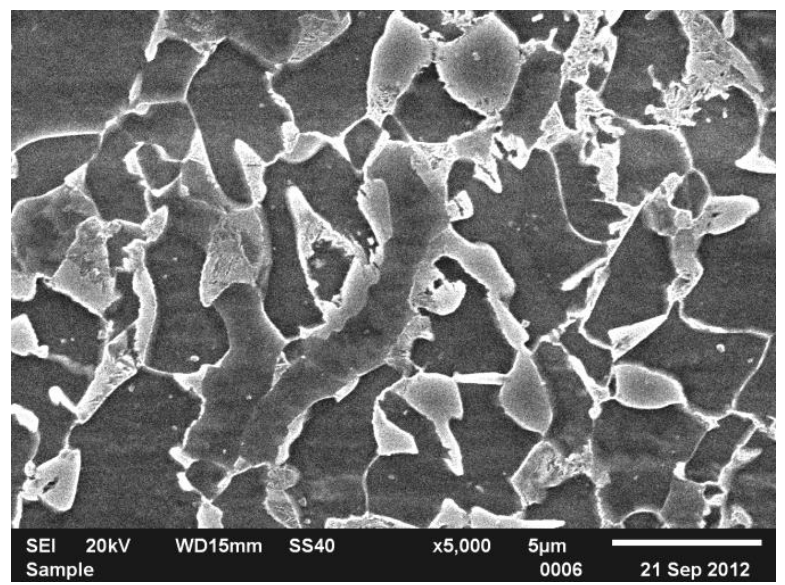

Figure 4. Small pearlite areas in the TRIP microstructure of CMnAlSi steel after continuous cooling from $800^{\circ} \mathrm{C}$ at $5^{\circ} \mathrm{C} / \mathrm{s}$.

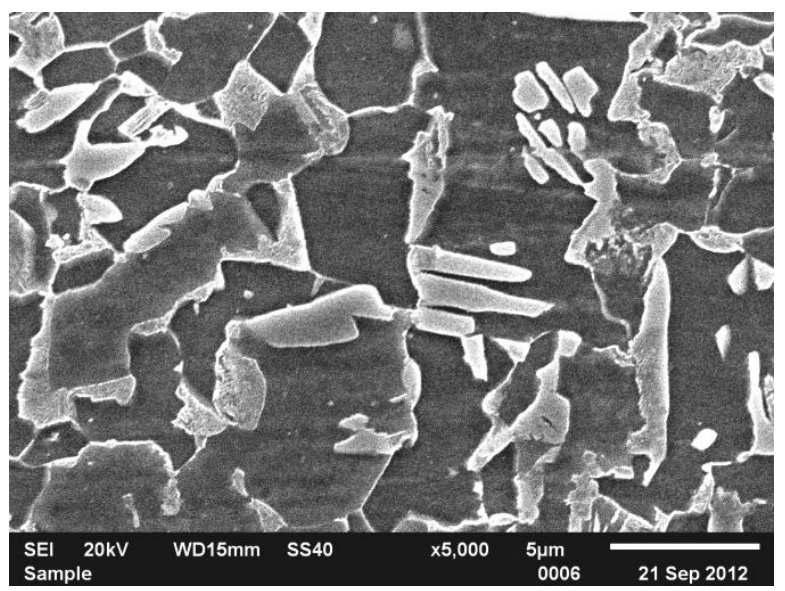

Figure 5. Martensite islands in the TRIP microstructure of CMnAlSi steel after continuous cooling from $800^{\circ} \mathrm{C}$ at $5^{\circ} \mathrm{C} / \mathrm{s}$.

Atomic Force Microscopy was used to study the same microstructure. The white areas in Figure 6 a) correspond to the bainitic ferrite and martensite lathes while dark in the image correspond to ferrite. The gray area in Figure 6 a) correspond to the austenite phase. The topographic study of this area Figure 6 b) shows three zones identified by differences in height, ferrite (low) retained austenite (medium) and bainitic ferrite (high).

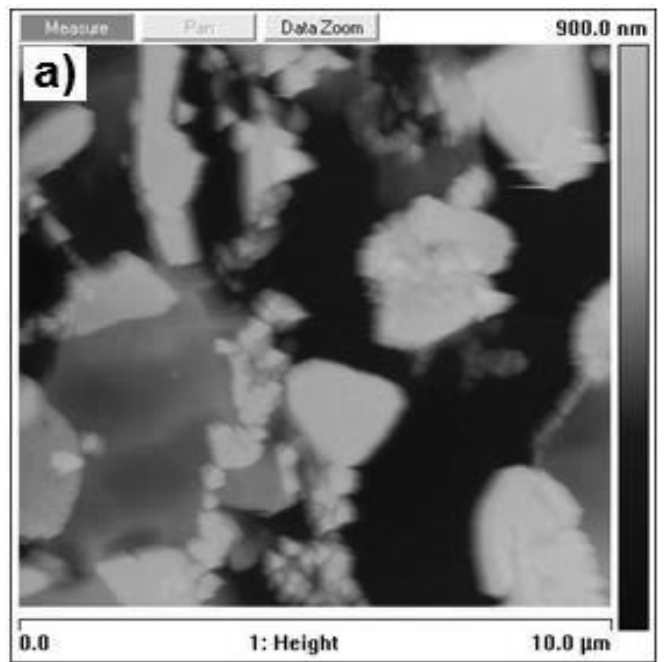

Figure 6a. Image in Height of TRIP steel microstructure from AFM

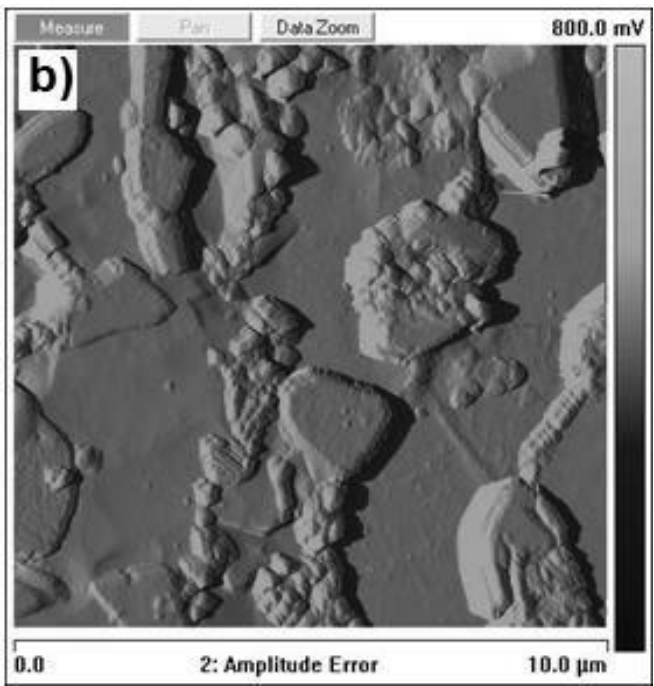

Figure 6b. Image in Amplitude Error of TRIP steel microstructure from AFM.

From topographical analysis for different levels of height with Atomic Force Microscopy (Figure 7) it was possible to identify the existing phases in CMnAlSi TRIP steel. The grain size of each phase of the microstructure is very small. The mean grain size of bainite and residual austenite vary between 1 and $3 \mu \mathrm{m}$, while that of the ferrite grains varies between 3 and $10 \mu \mathrm{m}$. 


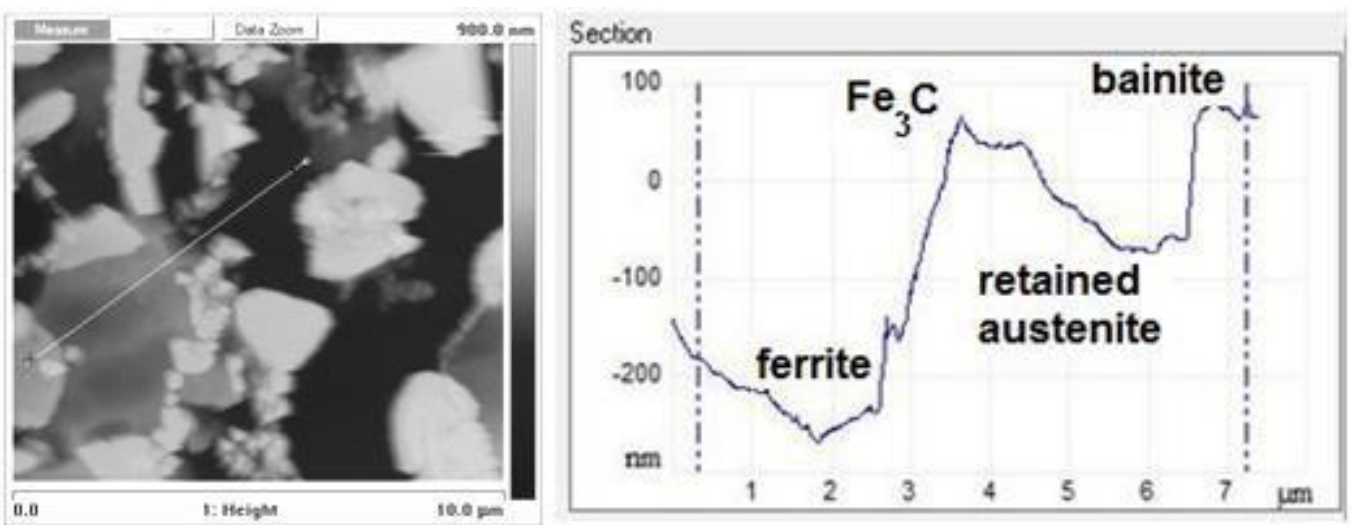

Figure 7. Height profile line to analyze the surface features and identify the phases in the multiphase CMnAlSi steel, field of view $10 \times 10 \mu \mathrm{m}^{2}$, cooled sample at rate from $800^{\circ} \mathrm{C}$ with $\mathrm{V}=5^{\circ} \mathrm{C} / \mathrm{s}$

Deep etching in nital allowed for better isolation of ferrite compared to other structural components. Thus areas of ferrite placement are easier to identify because they are deeper compared to other structural components [2].

\section{CONCLUSION}

AFM has been used to detailed characterization of the microstructure of CMnAlSi TRIP steel.

\section{REFERENCES}

[1] Ros-Yanez T., Houbeart Y., Martens A.,: Characterization of TRIPassisted multiphase surface topography by atomic force microscopy, Materials Characterization 47, 2001, 93-104

[2] Dryja M., Lis A., Wieczorek P.: TRIP steel topography made using AFM (Atomic Force Microscopy) Journal of Material Science (in press).

Magda Dryja defended her thesis titled: The microstructure and properties of steel plates $\mathrm{C} 15 \mathrm{~V}$ " in 2009 . He is currently a $\mathrm{PhD}$ student at the Technical University of Czestochowa. He has 18 publications in Polish and foreign languages. She has participated in numerous conferences and symposiums, which presented the results of the work. His work deals mainly with the controlled production of TRIP steel structure CMnAlSi.

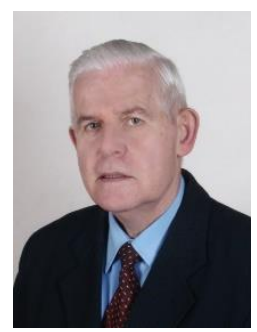

Prof. Andrzej Lis is a Professor of Physical Metallurgy and Materials Science and Engineering in Institute of Materials Engineering of Częstochowa University of Technology, Poland. 